\title{
EL EMPLEO Y LA INVERSIÓN EN LA INDUSTRIA ALICANTINA*
}

\author{
Rafael Sebastiá Alcaraz
}

\section{RESUMEN}

Los actuales paisajes industriales en la provincia de Alicante son el resultado de distintas formas de organizar la producción a lo largo de más de dos siglos. En el período estudiado, 1970-1991, se ha producido el fin de un modelo y la aparición de otro. La modificación de la estructura industrial ha afectado al empleo, con lo que la población y el territorio se han visto alterados.

Palabras claves: Empleo, inversión y territorio en Alicante.

\begin{abstract}
The present industrial landscapes in the province of Alicante are the result of different ways of organizing the production throughout more than two centuries. During the studied period, 1970-1991, the end of a model and the beginning of another one have been produced. The modification of the industrial structure has affected employment; thus, both population and territory have been altered.
\end{abstract}

Key words: Employment, investment and territory in Alicante.

\section{Introducción}

Producciones como la del hierro, carbón o ácido sulfúrico han sido consideradas habitualmente como indicadores del grado de industrialización de un territorio, hasta que la evolución industrial los ha relegado por su impacto ambiental, contaminación, etc, a países en vías de desarrollo o al Tercer Mundo.

Para el caso de la provincia de Alicante se ha venido observando que los tradicionales parámetros de industrialización no eran aplicables, porque el desarrollo industrial se había gestado no sobre una industria pesada, sino sobre una de bienes de consumo. Durante

* Investigación realizada dentro del Proyecto G.V. 2401/94. 
mucho tiempo, como un punto de referencia, se ha considerado, entre otros valores, el porcentaje de población ocupada o activa.

Sin embargo, en la década de los noventa no se puede considerar el empleo como el mejor indicador de la industrialización provincial, pues con el cambio de modelo industrial no resulta válido. Los gerentes industriales han procedido a modernizar los equipamientos, con el objetivo de aumentar la producción y la productividad, al mismo tiempo que reducían los puestos de trabajo. El nuevo modelo ha requerido mayores servicios y menos empleo directo en las factorías, con lo que el potencial indicador de personas trabajando directamente en los establecimientos ha dejado de ser significativo.

En esta evolución no se debe perder de vista, por un lado, que no siempre el cambio se ha realizado dentro de la legalidad ${ }^{1}$, y por otro que la modernización del equipamiento no se puede desconectar de la inversión. La evolución de la inversión y del empleo quedan, por tanto, estrechamente unidas.

\section{Evolución y distribución de la población activa}

La población activa de la provincia entre 1975 y 1991, según los datos de la E.P.A. y considerando el cambio estadístico recogido entre 1979 y 1980, (Cuadro I), aumentó al igual que la población de derecho. El crecimiento relativo de dicha población en la provincia de Alicante fue mayor que el registrado en la Comunidad Valenciana o en España durante el mismo período, pues, la provincia pasó de representar el 31'8\% respecto a la primera y del $3 \%$ respecto a la segunda durante 1975 , al $34{ }^{\prime} 1 \%$ y al 3'4\%, respectivamente, en 1991.

La evolución de la distribución sectorial de la población activa provincial durante estos años tampoco se repartió por igual en todos los sectores económicos, (Cuadro II). Así, la población activa agrícola, en cifras absolutas, retrocedió desde 1975 a 1991 un 57\%, y pasó de representar el 14'6\% del total a sólo el 4’9\%. La población activa industrial, pese al descenso registrado con fondo en 1986, en 1991 tenía niveles absolutos ligeramente superiores a los de 1975, aunque su participación en el total de la población activa se redujo de un 35'8\% en 1975 a un 28'6\% en 1986. La población activa dedicada al sector de la construcción, aumentó tal y como indica el índice de variación (123'8\%); no obstante, su participación en el total de la población activa disminuyó ligeramente.

El sector económico que marcó la diferencia y que registró el mayor crecimiento en la población activa fue el de servicios, pues durante este período su cifra absoluta creció un 93\% y su participación en el total de la población activa pasó del 34'9\% en 1975, algo inferior al de la industria, al 50’9\% en 1991. Sin duda, estos valores suponen la aparición de una sociedad y de una distribución de las actividades bastante distinta a la descrita por algunos investigadores, que hablan de una provincia de Alicante claramente industrializada, porque casi la mitad de sus activos trabajaban en el sector industrial ${ }^{2}$. En 1991, la situación ha cambiado claramente, pues en la industria trabajaba sólo un poco más de la cuarta parte de la población activa, mientras que en los servicios lo hacía la mitad de la misma $\left(50{ }^{\prime} 9 \%\right)$. La transformación aún fue más profunda si se tienen en cuenta las

1 MORENO FONSERET, Roque: La crisis económica en la Provincia de Alicante, (1973-1983), Caja de Ahorros Provincial, Alicante, 1989.

2 «...la consolidación de una base industrial que absorbe con regularidad creciente a prácticamente el 50\% de la población activa provincial y que aporta un $40 \%$ del valor añadido de la zona, permite definir, en principio y con carácter general, a la provincia de Alicante como área industrial» Ybarra i Pérez, Josep-Antoni (Coordinador), Sector calzado presente y futuro (abril-1986), Banco de Alicante, p. 6, Alicante, 1986. 
Cuadro I

POBLACIÓN ACTIVA DE ALICANTE, COMUNIDAD VALENCIANA Y ESPAÑA (1975 A 1991)

\begin{tabular}{|c|c|c|c|c|c|c|c|c|c|}
\hline \multirow[b]{2}{*}{ Año } & \multicolumn{4}{|c|}{ Alicante } & \multicolumn{3}{|c|}{ Comunidad Valenciana } & \multicolumn{2}{|c|}{ España } \\
\hline & Total & (1) & (2) & (3) & Total & (3) & (1) & Total & (1) \\
\hline 1975 & 402,4 & 100,0 & 31,8 & 3,0 & $1.266,0$ & 9,5 & 100,0 & $13.355,0$ & 100,0 \\
\hline 1976 & 402,2 & 100,0 & 31,3 & 3,0 & $1.285,8$ & 9,7 & 101,6 & $13.215,5$ & 99,0 \\
\hline 1977 & 410,4 & 102,0 & 31,3 & 3,1 & $1.310,0$ & 9,9 & 103,5 & $13.247,5$ & 99,2 \\
\hline 1978 & 426,6 & 106,0 & 32,3 & 3,2 & $1.320,3$ & 10,0 & 104,3 & $13.164,6$ & 98,6 \\
\hline 1979 & 453,7 & 112,7 & 33,2 & 3,4 & $1.368,3$ & 10,4 & 108,1 & $13.155,2$ & 98,5 \\
\hline 1980 & 428,7 & 106,5 & 33,0 & 3,3 & $1.299,6$ & 10,1 & 102,7 & $12.860,2$ & 96,3 \\
\hline 1981 & 448,3 & 111,4 & 33,5 & 3,5 & $1.336,8$ & 10,3 & 105,6 & $12.918,9$ & 96,7 \\
\hline 1982 & 452,4 & 112,4 & 33,2 & 3,5 & $1.364,0$ & 10,4 & 107,7 & $13.101,1$ & 98,1 \\
\hline 1983 & 456,0 & 113,3 & 33,5 & 3,5 & $1.359,6$ & 10,3 & 107,4 & $13.210,1$ & 98,9 \\
\hline 1984 & 481,3 & 119,6 & 34,7 & 3,6 & $1.386,6$ & 10,5 & 109,5 & $13.228,7$ & 99,1 \\
\hline 1985 & 475,7 & 118,2 & 34,5 & 3,6 & $1.379,8$ & 10,3 & 109,0 & $13.345,5$ & 99,9 \\
\hline 1986 & 449,5 & 111,7 & 33,2 & 3,2 & $1.355,6$ & 9,6 & 107,1 & $14.052,6$ & 105,2 \\
\hline 1987 & 493,2 & 122,6 & 34,2 & 3,4 & $1.441,9$ & 9,9 & 113,9 & $14.497,8$ & 108,6 \\
\hline 1988 & 492,2 & 122,3 & 34,2 & 3,4 & $1.437,7$ & 9,8 & 113,6 & $14.620,7$ & 109,5 \\
\hline 1989 & 499,8 & 124,2 & 33,7 & 3,3 & $1.484,4$ & 9,9 & 117,3 & $14.929,9$ & 111,8 \\
\hline 1990 & 537,1 & 133,5 & 35,2 & 3,6 & $1.526,1$ & 10,1 & 120,5 & $15.044,1$ & 112,6 \\
\hline 1991 & 518,2 & 128,8 & 34,1 & 3,4 & $1.521,8$ & 10,1 & 120,2 & $15.125,1$ & 113,3 \\
\hline
\end{tabular}

FUENTE: E.P.A., principales resultados (Cuarto Trimestre)

Hasta 1979 se incluye la población de 14 a 65 años; a partir de 1980 la población con más de 16 años.

Totales en miles de personas

(1) Índice de variación

(2) Porcentaje respecto al total de la Comunidad Valenciana

(3) Porcentaje respecto al total de España

tendencias, pues mientras el sector secundario resultó recesivo, el terciario estaba en expansión.

La tendencia evolutiva de la distribución de la población activa ha sido similar a la que siguió, con algunas diferencias, el conjunto del Estado. En España cabe señalar que el porcentaje de población activa agraria es superior al de Alicante en 1991, 10’7\% y 4’9\%, respectivamente. La población activa industrial también ofrece algunas diferencias significativas; por un lado, mientras en Alicante se ha sostenido el índice de variación, en España ha retrocedido; por otro lado, la participación de la población activa industrial en el total de la misma siempre ha sido inferior a la de la provincia.

La población activa de servicios presenta características más homogéneas en los dos ámbitos indicados; esto contribuye a reforzar la trayectoria seguida y la de los cambios económicos acontecidos. De esta forma, en España, la población activa de servicios en 1991 supuso el 51'6\% de la población activa total y en Alicante, el 50'9\%.

Por tanto, se puede concluir que la evolución de la población activa en todos los espacios considerados, y con mayor intensidad en la provincia de Alicante, señala por un lado, que el retroceso de la población activa en la agricultura no ha cesado y por otro, que existe un desplazamiento desde el sector agrario e industrial hacia el de servicios. 


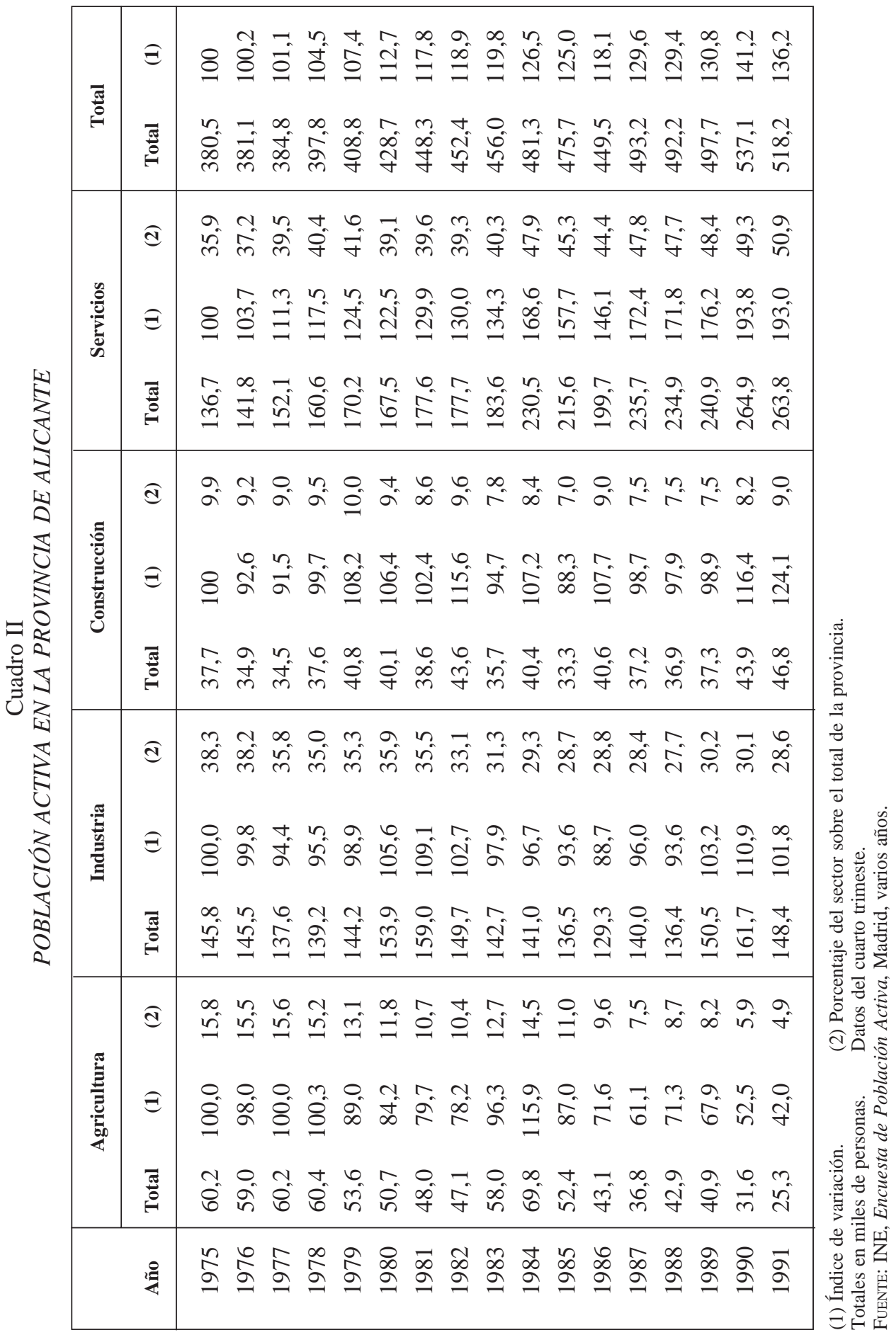




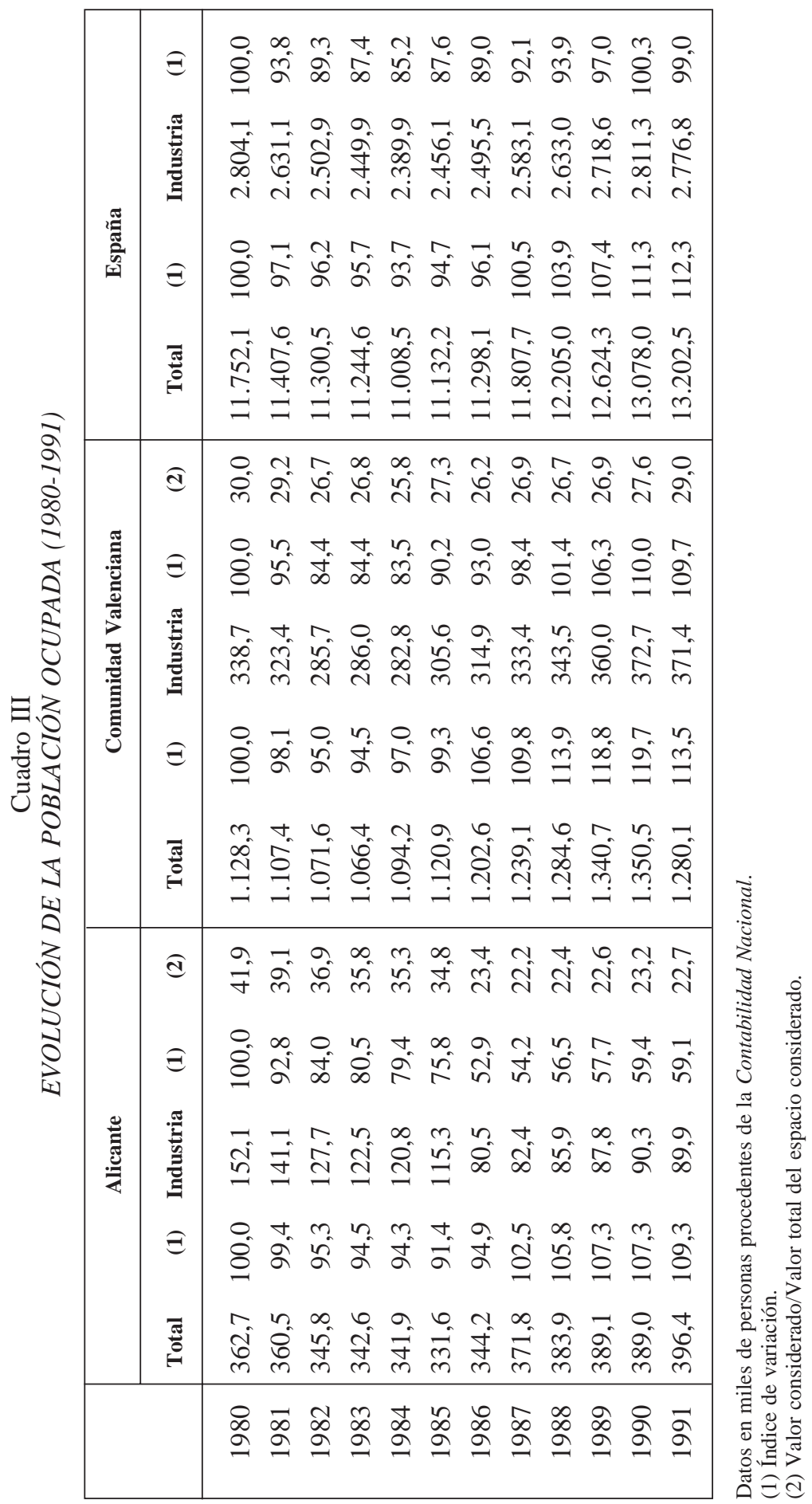




\section{Evolución y distribución de la población ocupada}

La evolución y distribución de la población ocupada en España presenta características similares a las descritas en la población activa: disminución del empleo tanto en cifras absolutas como relativas en los sectores agrario e industrial, estabilidad en el de la construcción y una fuerte expansión en el de servicios.

El aspecto más sobresaliente lo constituye la evolución del total de la población ocupada entre 1980 y 1991, aunque con dos períodos claramente diferenciados: En el primero (1980-84) se asiste a un retroceso de la población ocupada total, y en el segundo (1985-91) a una recuperación (Cuadro III). La causa de esta oscilación fue principalmente la evolución del sector industrial y de la construcción, pues mientras la población ocupada en la agricultura presentó una tendencia descendente, la de los servicios aumentó; la evolución de la industria y de la construcción, a partir de 1988 y 1987, respectivamente, modificaron su comportamiento pasando de un ciclo regresivo a otro de expansión.

La diferencia entre población activa y ocupada corresponde obviamente a la población parada y la consecuencia socialmente más nefasta ha sido el fuerte incremento del paro. No obstante, desde 1987 aparece un aspecto positivo: la evolución de la población ocupada creció más rápidamente que la de la población activa y por tanto el paro tendió a reducirse.

La población ocupada de Alicante, comparada con la de la Comunidad Valenciana y la de España, desde 1980 ha presentado aspectos diferentes. En primer lugar, en Alicante la población total ocupada aumentó menos que en la Comunidad Valenciana o en España, y cuando se recuperó, no lo hizo tan tempranamente como en los dos casos indicados. En segundo lugar, la población ocupada en la industria ha presentado una tendencia fuertemente regresiva que contrasta con el índice positivo de la Comunidad Valenciana e incluso con la estabilidad mantenida por la del conjunto de España. En tercer lugar, la población ocupada industrial de Alicante en 1991, presenta valores sobre la población ocupada total $(22,7 \%)$ similares a los de España $(21,0 \%)$, mientras que el conjunto de la Comunidad Valenciana aparece más definido por esta actividad (29,0\%).

Por tanto, se puede concluir que durante la década de los ochenta e inicios de los noventa, la población ocupada de Alicante presenta una clara crisis en el sector industrial, el cual, hasta 1980, definía la estructura económica y social de la provincia. Este cambio, sin duda, se debe relacionar más con la reestructuración industrial que con una desindustrialización, ya que la producción, la productividad y la competitividad iban en aumento.

De un análisis sectorial de la población ocupada industrial en la provincia de Alicante, se puede concluir que:

- La población ocupada se concentró en el sector calzado, al igual que la producción, superando siempre más del 30\% del empleo. Por otro lado el sector de la construcción era el segundo en cuanto a población ocupada.

- El resto de los sectores, excepto el de transformados metálicos, en 1971 no superaban el 10\% de la población ocupada. Por encima del 5\% estaban el anterior, el de alimentación, bebidas y tabaco, el textil y el químico, que entre 1971 y 1981 incluyó el del caucho y plásticos.

— Esta composición apenas varía entre 1971 y 1991.

En el conjunto de la Comunidad Valenciana la distribución de la población ocupada industrial presentó unas características diferentes:

En primer lugar, la población ocupada no se concentró en la misma medida en ningún sector industrial; por esta razón no se puede hablar de dependencia económica respecto a 
una determinada actividad industrial. En este caso, es el sector de la construcción el que más empleo generó seguido por el del calzado. El sector transformados metálicos, que ocupaba la tercera posición, redujo su importancia como se puede observar con los datos de 1991.

En segundo lugar, es notoria la trascendencia de la industria del calzado, pues aunque se halla fuertemente implantada en pocas comarcas alicantinas, alcanzó un porcentaje significativo dentro de la Comunidad Valenciana.

En tercer lugar, superando el 5\% de la población ocupada industrial, existían más sectores industriales que en Alicante: el de la alimentación, textil, madera y mueble, química, cerámica vidrio y cemento, y transformados metálicos.

Por tanto, se puede concluir que la industria de la Comunidad Valenciana ofreció una mayor diversificación que la de Alicante, donde la concentración en unos pocos sectores, y en concreto, en el calzado fue decisiva.

La distribución de la población ocupada comparada con la del total del Estado resultó asimismo diferente, y de una forma global se puede afirmar que la provincia de Alicante presentó particularidades en la distribución de la población ocupada que han conducido, junto con la interpretación de otros factores, a la afirmación de un modelo industrial específico.

El crecimiento demográfico de la provincia no se ha visto acompañado de un incremento del empleo, y menos del empleo industrial, progresivamente reducido. La disminución del empleo ha sido consecuencia, entre otras causas, de la modernización de los equipos productivos y no ha influido en un descenso de la productividad industrial, que por el contrario ha ido en aumento. Por esta causa, la provincia corre el riesgo, de no mediar ninguna intervención pública, que en un futuro bastante inmediato los municipios exclusivamente industriales sufran despoblación progresiva, como antaño ocurrió con los agrícolas.

\section{El empleo en Europa}

La evolución del empleo en la CEE, entre 1980 y 1987, también siguió una trayectoria opuesta a la producción y, al igual que ocurrió en la provincia de Alicante, el número total de empleos en la industria se fue reduciendo. Pero no ocurría en todas partes igual, pues en los Estados Unidos el empleo incluso llegó a aumentar.

Por sectores, en la CEE el empleo se redujo en la agricultura y en la industria, y se incrementó en los servicios ${ }^{3}$. La evolución positiva del empleo en servicios y la recesión del empleo en industria, confirman la tesis de una Europa postindustrial, donde las empresas industriales generan más valor añadido y empleo a través de los Inputs procedentes de servicios prestados, que de los obtenidos en los tradicionales «procesos productivos». La diferenciación entre servicios destinados a ventas y los que no lo están, permite comprobar esta evolución. Dentro de la industria existe un mayor reparto del empleo entre los distintos sectores, pero continúan destacando las actividades manufactureras por excelencia como el del textil y vestidos, el cuero y el calzado. La evolución del empleo en estas actividades también presenta en la CEE una trayectoria regresiva, de modo que lo acontecido con el empleo en la provincia de Alicante, con un sector que resulta hegemónico, se corresponde con una tendencia general de la Europa Occidental. Por tanto, queda claro que la localiza-

3 Colin Clark señaló que el progreso económico se hallaba vinculado a un desarrollo de los servicios, aunque posteriormente tal afirmación se vincula fundamentalmente al terciario superior. 
ción geográfica de la industria alicantina obedece a unas razones de ámbito superior al estrictamente provincial.

El siguiente sector en importancia por el empleo que genera, dentro de la CEE, es el de la alimentación, bebidas y tabaco. En este caso se realizan dos observaciones: la primera es resaltar que los dos sectores que más empleo crean en la CEE se hallan ampliamente consolidados en el espacio alicantino, con las ventajas sociales que ello implica, y la segunda es destacar que el sector de la alimentación, bebidas y tabaco, fue, aunque en menor medida, un sector regresivo desde el punto de vista del empleo.

Del resto de los sectores, sobresale el denominado medios de transporte, con escasa implantación en Alicante, pero sí en la Comunidad Valenciana. La construcción de maquinaria y similares, deficitaria en Alicante, se halla por el contrario más representada por el empleo en la CEE. La industria del juguete, especialmente localizada en la Hoya de Castalla, comparada con su similar productos de caucho y plástico no resulta ser uno de los sectores que más trabajo genera en en la CEE ya que éste se halla estancado. El sector de los minerales y productos no metálicos, que ofrece una distribución más generalizada en la provincia de Alicante, tampoco destaca en el marco de la CEE con una evolución igualmente descendente.

A partir de 1988, como señala la Comisión ${ }^{4}$, se entra en una fase en la que la inversión no repercute en un crecimiento del empleo. Los sectores más afectados fueron tanto los innovadores, material informático y electrónica, como los tradicionales, vulnerables a la competencia internacional y a la evolución de la construcción, textil-hogar, madera y mueble; todos ellos de amplia implantación en la Comunidad Valenciana. Las empresas de estos sectores intentaron competir aumentando la productividad. Otro sector, no significativo en la provincia, pero que recogió una drástica reducción, al igual que el sector agrícola, fue el siderúrgico. En conjunto, la tasa media de crecimiento del empleo en la CEE pasó del 1,7\% en 1988 al 0,5\% en 1991, mostrando una vez más la tendencia al estancamiento en la creación de puestos de trabajo en la CEE.

Por tanto, se puede afirmar y resumir, que la provincia de Alicante se benefició del desarrollo de los dos sectores industriales que más empleo generaron en la CEE, aunque la evolución del empleo en los sectores predominantes de Alicante no fue positiva, coincidiendo con lo acontecido en la CEE.

\section{Empleo industrial previsto 5}

El empleo industrial previsto depende de una serie de circunstancias, como son: la productividad y competitividad de las empresas, el desarrollo tecnológico de los equipos productivos, las expectativas de la demanda, etc. En este apartado no se pretende analizar las causas que lo han producido, sino su distribución y su evolución sectorial.

En el análisis del empleo industrial previsto, en primer lugar se considerará la trayectoria de su evolución y el tipo de inversión que ha influido más en la creación del mismo. Posteriormente se estudiará los sectores industriales en los que se ha producido más creación de empleo.

El empleo industrial previsto, desde 1973 a 1991 ha presentado un continuo retroceso tanto en la Comunidad Valenciana como en Alicante, (Cuadro IV). Este descenso, tal y

4 «Las inversiones realizadas durante estos últimos años deberían garantizar tasas de crecimiento positivas de la productividad y del empleo, pero un crecimiento del empleo en sí mismo resulta poco probable» Comisión de las Comunidades Europeas, Panorama de la industria comunitaria, 1991-1992, Luxemburgo, 1991, p. 38.

5 El empleo previsto se refiere a los puestos de trabajo que se preveen crear cuando se realizan las inscripciones de inversión en el Registro Industrial. 


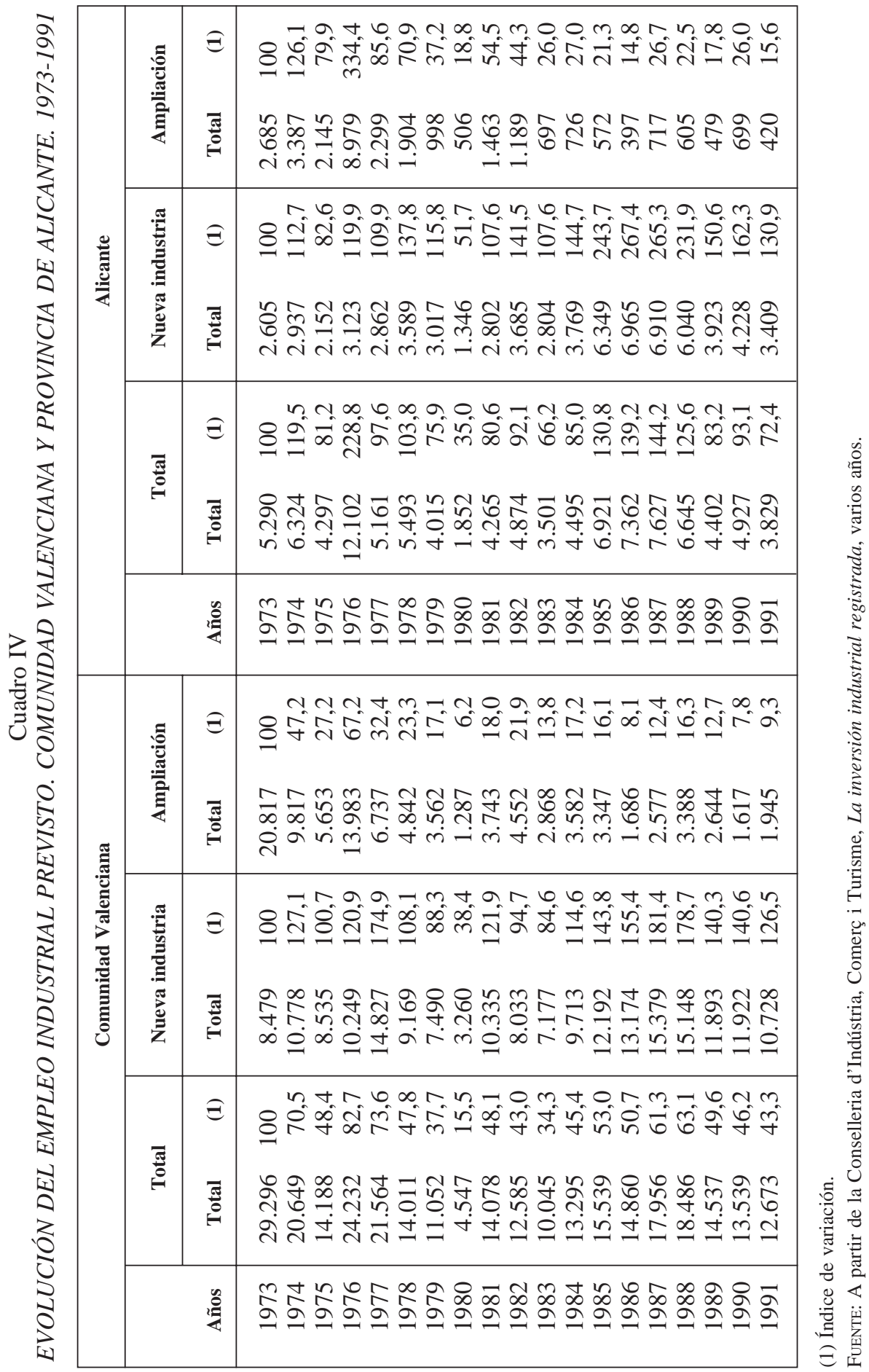


como señala el índice de variación, ha sido más intenso en la Comunidad Valenciana $(43,3 \%)$, que en la provincia de Alicante $(72,4 \%)$. La creación de empleo industrial ha correspondido, en los dos espacios analizados, en mayor medida a la creación de nuevos establecimientos industriales, que a la ampliación. Durante este período se observan dos etapas bien diferenciadas: la primera corresponde al periodo 1973-1976 en el que existe un equilibrio favorable en la creación de empleo por parte de las industrias que se amplían; la segunda, empieza en 1977 y la génesis de empleo pasa a depender en su mayor parte de la nueva industria.

La recesión en el empleo previsto, tanto en Alicante como en el conjunto de la Comunidad Valenciana, procede de la ampliación de las industrias y no de las nuevas inscripciones, en las que se aumentaba el mismo (Cuadro IV).

Por tanto, la crisis y los cambios en el empleo industrial previsto, inducen a afirmar que la crisis de mediados de los setenta supuso el fin de un modelo industrial. Hasta esta fecha el sistema adoptado por las empresas era el de continuadas ampliaciones. A partir de 1977 se introduce otro modelo, relacionado con la potenciación de las pequeñas y medianas instalaciones y que por distintas razones (descentralización de la producción, ayudas administrativas) se prolonga hasta 1991.

La evolución del empleo previsto y del total de la población ocupada en la industria desde 1980 (fecha desde la que se pueden relacionar las dos variables) están estrechamente unidas y sus trayectorias confirman la validez de la información. La recesión del empleo previsto, como era previsible, iba a influir en un retroceso de la población ocupada. La diferencia se encuentra en que el empleo previsto en Alicante ha retrocedido menos que en el conjunto de la Autonomía a la que pertenece, y en cambio la población ocupada ha disminuido más. Una posible explicación se encuentra en que en Alicante se han cerrado comparativamente más empresas que en la Comunidad Valenciana; por tanto, aunque en Alicante se creen más puestos de trabajo, el cierre de un mayor número de empresas contribuye a que descienda la población ocupada. Esto no significa que en la provincia existan más personas desocupadas, pues el desarrollo de la economía sumergida, en especial en una actividad tan ampliamente consolidada como la del sector calzado, puede favorecer la ocultación.

\section{Empleo previsto por sectores industriales en la provincia de Alicante}

La evolución del empleo previsto tampoco ha sido uniforme dentro de la provincia de Alicante y ha variado por sectores. Se puede afirmar, desde este punto de vista, que la estructura industrial, y por tanto su impacto sobre el espacio provincial, ha variado con el tiempo.

En primer lugar es destacable el sector calzado por la capacidad de generar empleo previsto. En 1975 ocupaba la segunda posición tras la industria textil y en 1991 pasó a ocupar la primera, (Cuadro V). La evolución resulta más significativa si se considera que en 1975 creaba el 19'1\% del empleo industrial previsto en la provincia y en 1991 el $42 \%$.

En segundo lugar se observa una alternancia en los sectores de «arrastre» de la provincia, pues en 1975 la industria textil era la que más empleo generaba, el 22’1\%, mientras en 1991 tan sólo generaba el 8\%. Este cambio se debe al desarrollo de otras industrias, a la regresión implícita del sector y a una importante reducción de mano de obra como consecuencia de la introducción de nuevas tecnologías.

Otros sectores que han perdido protagonismo (en el empleo previsto y entre las mismas fechas) han sido el de la alimentación, que ha pasado del 9,3\% al 4,5\%, el de la construc- 


\section{Cuadro $\mathrm{V}$}

\section{INVERSIÓN Y EMPLEO PREVISTO EN LA PROVINCIA DE ALICANTE POR SECTORES INDUSTRIALES. 1975 Y 1991}

\begin{tabular}{|c|c|c|c|c|c|c|c|c|c|c|c|c|}
\hline \multirow[b]{3}{*}{ Sectores } & \multicolumn{8}{|c|}{1975} & & & & \\
\hline & \multicolumn{4}{|c|}{ Nueva Industria } & \multicolumn{4}{|c|}{ Ampliación } & \multicolumn{4}{|c|}{ Total } \\
\hline & Inversión & Empleo & (1) & (2) & Inversión & Empleo & (1) & (2) & Inversión & Empleo & (1) & (2) \\
\hline Energía y agua & 0 & 0 & 0 & - & 0 & 0 & 0 & - & 0 & 0 & 0,0 & - \\
\hline Producción y primera transformación de metales & 0 & 0 & 0 & - & 35.386 & 18 & 0,8 & 1.966 & 35.386 & 18 & 0,4 & 1.966 \\
\hline Extracción materiales de construcción & 0 & 0 & 0 & - & 0 & 0 & 0 & - & 0 & 0 & 0,0 & - \\
\hline Fabricación materiales de construcción & 57.002 & 80 & 3,7 & 713 & 47.493 & 28 & 1,3 & 1.696 & 104.495 & 108 & 2,5 & 968 \\
\hline Industria piedra natural & 15.392 & 25 & 1,2 & 616 & 33.997 & 23 & 1,1 & 1.478 & 49.389 & 48 & 1,1 & 1.029 \\
\hline Vidrio y cerámica & 525 & 3 & 0,1 & 175 & 10.515 & 0 & 0,0 & 0 & 11.040 & 3 & 0,1 & 3.680 \\
\hline Industría química & 4.847 & 19 & 0,9 & 255 & 21.765 & 60 & 2,8 & 363 & 26.612 & 79 & 1,8 & 337 \\
\hline Fabricación productos metálicos & 5.968 & 45 & 2,1 & 133 & 224.998 & 54 & 2,5 & 4.167 & 230.966 & 99 & 2,3 & 2.333 \\
\hline Construcción maquinaria y equipos mecánicos & 16.382 & 118 & 5,5 & 139 & 27.477 & 46 & 2,1 & 597 & 43.859 & 164 & 3,8 & 267 \\
\hline Construcción maquinaria y mat. eléctrico y electrónico & 2.820 & 21 & 1,0 & 134 & 609 & 3 & 0,1 & 203 & 3.429 & 24 & 0,6 & 143 \\
\hline Construcción material transporte & 66.734 & 124 & 5,8 & 538 & 13.194 & 157 & 7,3 & 84 & 79.928 & 281 & 6,5 & 284 \\
\hline Instrumentos de precisión óptica & 0 & 0 & 0 & - & 0 & 0 & 0 & - & 0 & 0 & 0,0 & - \\
\hline Alimentación & 253.221 & 378 & 17,7 & 670 & 25.379 & 23 & 1,1 & 1.103 & 278.600 & 401 & 9,3 & 695 \\
\hline Bebidas y tabaco & 0 & 0 & 0,0 & 0 & 6.840 & 14 & 0,6 & 489 & 6.840 & 14 & 0,3 & 489 \\
\hline Textil & 265.051 & 293 & 13,7 & 905 & 446.665 & 657 & 30,4 & 680 & 711.716 & 950 & 22,1 & 749 \\
\hline Cuero & 8.365 & 59 & 2,8 & 142 & 6.601 & 77 & 3,6 & 86 & 14.966 & 136 & 3,2 & 110 \\
\hline Calzado & 68.764 & 475 & 22,2 & 145 & 54.046 & 346 & 16,0 & 156 & 122.810 & 821 & 19,1 & 150 \\
\hline Confecciones textiles & 31.920 & 143 & 6,7 & 223 & 15.999 & 43 & 2,0 & 372 & 47.919 & 186 & 4,3 & 258 \\
\hline Madera & 18.531 & 112 & 5,2 & 165 & 16.543 & 24 & 1,1 & 689 & 35.074 & 136 & 3,2 & 258 \\
\hline Muebles de madera & 6.919 & 58 & 2,7 & 119 & 9.998 & 29 & 1,3 & 345 & 16.917 & 87 & 2,0 & 194 \\
\hline Papel & 730 & 2 & 0,1 & 365 & 207.847 & 52 & 2,4 & 3.997 & 208.577 & 54 & 1,3 & 3.863 \\
\hline Artes gráficas y edición & 0 & 0 & 0 & - & 712 & 2 & 0,1 & 356 & 712 & 2 & 0,0 & 356 \\
\hline Caucho y plásticos & 53.357 & 130 & 6,1 & 410 & 101.770 & 233 & 10,8 & 437 & 155.127 & 363 & 8,4 & 427 \\
\hline Joyería y bisutería & 82 & 1 & 0,0 & 82 & 0 & 0 & 0 & - & 82 & 1 & 0,0 & 82 \\
\hline Juguetes & 4.485 & 26 & 1,2 & 173 & 132.112 & 248 & 11,5 & 533 & 136.597 & 274 & 6,4 & 499 \\
\hline Industrias manufactureras diversas & 1.123 & 24 & 1,1 & 47 & 1.095 & 24 & 1,1 & 46 & 2.218 & 48 & 1,1 & 46 \\
\hline \multirow[t]{4}{*}{ Total } & 882.218 & 2.136 & 100 & 413 & 1.441 .041 & 2.161 & 100 & 667 & 2.323 .259 & 4.297 & 100 & 541 \\
\hline & \multicolumn{12}{|c|}{1991} \\
\hline & \multicolumn{4}{|c|}{ Nueva Industria } & \multicolumn{4}{|c|}{ Ampliación } & \multicolumn{4}{|c|}{ Total } \\
\hline & Inversión & Empleo & (1) & (2) & Inversión & Empleo & (1) & (2) & Inversión & Empleo & (1) & (2) \\
\hline Energía y agua & 1.044 .627 & 65 & 1,9 & 16.071 & $12.221,0$ & 0 & 0 & $*$ & 1.056 .848 & 65 & 1,7 & 16.259 \\
\hline Producción y primera transformación de metales & 44.007 & 23 & 0,7 & 1.913 & $21.410,0$ & 3 & 0,7 & 7.137 & 65.417 & 26 & 0,7 & 2.516 \\
\hline Extracción materiales de construcción & 180.680 & 65 & 1,9 & 2.780 & $134.139,0$ & 31 & 7,4 & 4.327 & 314.819 & 96 & 2,5 & 3.279 \\
\hline Fabricación materiales de construcción & 390.078 & 85 & 2,5 & 4.589 & $158.083,0$ & 2 & 0,5 & 79.042 & 548.161 & 87 & 2,3 & 6.301 \\
\hline Industria piedra natural & 327.444 & 101 & 3,0 & 3.242 & $128.486,0$ & 25 & 6,0 & 5.139 & 455.930 & 126 & 3,3 & 3.618 \\
\hline Vidrio y cerámica & 22.543 & 13 & 0,4 & 1.734 & 0 & 0 & 0 & - & 22.543 & 13 & 0,3 & 1.734 \\
\hline Industria química & 72.341 & 49 & 1,4 & 1.476 & $27.757,0$ & 7 & 1,7 & 3.965 & 100.098 & 56 & 1,5 & 1.787 \\
\hline Fabricación productos metálicos & 438.213 & 256 & 7,5 & 1.712 & $147.001,0$ & 17 & 4,1 & 8.647 & 585.214 & 273 & 7,1 & 2.144 \\
\hline Construcción maquinaria y equipos mecánicos & 106.343 & 61 & 1,8 & 1.743 & 0 & 0 & 0,0 & - & 106.343 & 61 & 1,6 & 1.743 \\
\hline Construcción maquinaria y mat. eléctrico y electrónico & 35.223 & 47 & 1,4 & 749 & $4.534,0$ & 10 & 2,4 & 453 & 39.757 & 57 & 1,5 & 697 \\
\hline Construcción material transporte & 33.555 & 28 & 0,8 & 1.198 & $1.276,0$ & 0 & 0 & 0 & 34.831 & 28 & 0,7 & 1.244 \\
\hline Instrumentos de precisión óptica & 4.300 & 1 & 0 & 4.300 & 123,0 & 1 & 0,2 & 123 & 4.423 & 2 & 0,1 & 2.212 \\
\hline Alimentación & 272.389 & 147 & 4,3 & 1.853 & $157.397,0$ & 26 & 6,2 & 6.054 & 429.786 & 173 & 4,5 & 2.484 \\
\hline Bebidas y tabaco & 1.650 & 0 & 0 & $*$ & $187.197,0$ & 20 & 4,8 & 9.360 & 188.847 & 20 & 0 & 9.442 \\
\hline Textil & 418.167 & 267 & 7,8 & 1.566 & $376.387,0$ & 39 & 9,3 & 9.651 & 794.554 & 306 & 8,0 & 2.597 \\
\hline Cuero & 40.733 & 38 & 1,1 & 1.072 & 0 & 0 & 0 & - & 40.733 & 38 & 1,0 & 1.072 \\
\hline Calzado & 947.682 & 1.561 & 45,8 & 607 & $56.751,0$ & 47 & 11,2 & 1.207 & 1.004 .433 & 1.608 & 42,0 & 625 \\
\hline Confecciones textiles & 97.122 & 104 & 3,0 & 934 & $63.924,0$ & 2 & 0,5 & 31.962 & 161.046 & 106 & 2,8 & 1.519 \\
\hline Madera & 478.146 & 191 & 5,6 & 2.503 & $37.177,0$ & 56 & 13,4 & 664 & 515.323 & 247 & 6,5 & 2.086 \\
\hline Muebles de madera & 179.703 & 102 & 3,0 & 1.762 & 0 & 0 & 0 & 0 & 179.703 & 102 & 2,7 & 1.762 \\
\hline Papel & 43.069 & 14 & 0,4 & 3.076 & $801.506,0$ & 89 & 21,2 & 9.006 & 844.575 & 103 & 2,7 & 8.200 \\
\hline Artes gráficas y edición & 90.157 & 57 & 1,7 & 1.582 & $29.337,0$ & 13 & 3,1 & 2.257 & 119.494 & 70 & 1,8 & 1.707 \\
\hline Caucho y plásticos & 197.594 & 112 & 3,3 & 1.764 & $297.091,0$ & 25 & 6,0 & 11.884 & 494.685 & 137 & 3,6 & 3.611 \\
\hline Joyería y bisutería & 6.052 & 4 & 0,1 & 1.513 & 0 & 0 & 0 & 0 & 6.052 & 4 & 0,1 & 1.513 \\
\hline Juguetes & 20.614 & 19 & 0,6 & 1.085 & $94.004,0$ & 6 & 1,4 & 15.667 & 114.618 & 25 & 0,7 & 4.585 \\
\hline Industrias manufactureras diversas & 0 & 0 & 0 & - & 0 & 0 & 0 & - & 0 & 0 & 0,0 & - \\
\hline Total & 5.492 .432 & 3.410 & 100 & 1.611 & $2.735 .801,0$ & 419 & 100 & 6.529 & 8.228 .233 & 3.829 & 100 & 2.149 \\
\hline
\end{tabular}

Fuente: A partir de listados de la Conselleria d'Indústria, Comerç i Turisme.

* Se invierte y no se genera empleo.

Miles de pesetas corrientes.
(1) \% sobre total del empleo.
(2) Inversión/ empleo. 
ción de material de transporte, del 6,5\% al 0,7\%, el de caucho y plástico, del 8,4\% al 3,6\% y el del juguete, que ha pasado del $6,4 \%$ al $0,7 \%$.

Por contra, entre los sectores emergentes destacan el de fabricación de productos metálicos (2'3\% y $7^{\prime} 1 \%$ ), el de la madera (3'2\% al 6'5\%), y en menor medida los de energía y agua, el de extracción de materiales para la construcción, piedra natural, material eléctrico y electrónico, artes gráficas y edición.

Por tanto, el análisis del empleo previsto por sectores, también permite afirmar la existencia de un cambio en el modelo industrial, tal y como se desprende de la concentración del empleo y de la alternancia de los sectores de «arrastre».

\section{Empleo previsto por sectores e inversión en la provincia de Alicante}

Un elemento decisivo en la generación de empleo es la inversión. El capital necesario para crear un puesto de trabajo puede favorecer o dificultar el desarrollo de un sector industrial y a su vez informa de la renovación de los equipamientos, con la que los gerentes pretenden aumentar la productividad, la calidad o el diseño, y en definitiva la competitividad. La inversión por puesto de trabajo previsto, por tanto, es un buen indicador del desarrollo industrial y de la estructura productiva.

La inversión por puesto de trabajo previsto en la provincia de Alicante, ofrece las siguientes características:

1.- Uno de los sectores que más empleo generó, el del calzado, tanto en 1975 como en 1991, se situó bastante por debajo de la media provincial. En 1975 la media de inversión por puesto de trabajo previsto en los sectores industriales era de 541.000 pts. y en el sector del calzado de tan sólo 150.000 pts.; en 1991 los valores ascendieron, respectivamente, a 2.149.000 pts. y 625.000 pts. Esto confirma la dependencia y la gran demanda de mano de obra, con la que además con una mínima inversión se asegura una rentabilidad. Estas características favorecen tanto su dispersión productiva, como espacial.

2.- No todos los sectores con una baja relacion inversión-empleo tuvieron el mismo desarrollo; es el caso de joyería y bisutería, la confección textil, muebles de madera o cuero.

3.- La inversión por puesto de trabajo superior a la media provincial puede facilitar información de sectores con características productivas específicas, en concreto de aquellos que requieren de equipamientos más costosos, como ocurre con la industria del papel o la textil y/o de fenómenos puntuales de reconversión.

En el primer caso, las mayores exigencias de capital favorecen la concentración industrial. Las manufacturas textil y papelera son un buen ejemplo de esta característica. Otras actividades similares pueden ser la de la piedra natural, fabricación de materiales de construcción, producción y primera transformación de metales. El estudio de la industria de la alimentación obliga a introducir una matización, pues aunque se trata de una industria con fuertes concentraciones espaciales y sectoriales, por ejemplo en Xixona o la Vila Joiosa, está ampliamente repartida por la provincia, entre otras razones por su dependencia de los mercados. Sin embargo, las necesidades productivas influyen en inversiones más cuantiosas que en la media de los sectores industriales.

En el segundo caso, la aparición de un nuevo modelo industrial, en el que se intenta reducir la incidencia de los costes de la mano de obra en el precio final de la producción, unido el desarrollo tecnológico y al incremento de la competencia, repercute en la necesidad de disponer de más capital por cada puesto de trabajo previsto. La industria del juguete o la del caucho y plásticos se hallan en esta situación

Por tanto, aunque el cociente inversión-empleo previsto dependa de la situación de 
cada sector, la concentración o dispersión de la industria guarda relación con las exigencias de capital por puesto de trabajo.

Como ya se ha dicho, el empleo previsto dependió del tipo de inversión, es decir, si se trata de la creación de una nueva industria o de una ampliación. En este sentido la inversión por trabajo previsto se ha caracterizado por:

a) Una disposición de menor capital por empleo tanto en 1975 como en 1991, en la creación de nuevas industrias. Esto supone que con menos dinero se crearon más puestos de trabajo en las nuevas industrias que en las ampliaciones.

b) La inversión en ampliación de las empresas o renovación del equipamiento se asocia a una reducción de la demanda de mano de obra.

c) La existencia de una diferencia substancial entre 1975 y 1991. En 1975 la inversiónempleo en ampliación, aunque más elevada que la correspondiente a la creación de nueva empresa, no presentaba una disparidad tan marcada como en 1991, (Cuadro V). Obviamente la situación se debió a modelos industriales diferentes. En la primera fecha, existía una industria que se había consolidado aprovechando la abundancia y los costes reducidos de la mano de $\mathrm{obra}^{6}$, y en la que el aumento de la producción se realizaba básicamente aumentando el número de empleados. En 1975, los establecimientos industriales disponían de unas plantillas mayores que en 1991, período en el que la industria tendió a reducir el número de empleados y a incrementar su productividad.

d) Esta diferencia entre nueva industria y ampliación se repite en casi todos los sectores. No obstante, puntualmente se producen «anomalías» por exceso o por defecto respecto a la situación media.

En efecto, en algunos sectores la inversión por puesto trabajo resulta más cara en la creación de una nueva industria que en su ampliación. Este es el caso de la industria textil en 1975, donde la inversión fue más elevada en nueva industria que en ampliación; se reproduce la misma situación en la industria de material eléctrico, electrónico, instrumentos de precisión y en la de la madera durante 1991. Como explicación se supone que el equipamiento o infraestructura inicial, según los casos, requiere más capital que el resto de los sectores y que las ampliaciones posteriores.

Por el contrario, una mayor diferencia respecto a la media, entre nueva industria y ampliación, puede indicar una renovación o reconversión de un sector industrial. Con esta situación destaca en 1975 la fabricación de productos metálicos, o la industria del papel, y en 1991 la de fabricación de materiales de construcción, confección textil, o la del juguete.

Por tanto, se puede establecer una relación significativa entre creación de empleo e inversión, ya que la inversión más reducida por puesto de trabajo en la nueva industria coincide con la mayor creación de éstos y, por el contrario una mayor inversión en las ampliaciones produce una reducción de los mismos. Del mismo modo, se puede concluir que los cambios de modelo industrial influyen en esta relación, y que la mayor parte de los sectores industriales recogen la tendencia general expuesta.

\section{Evolución del empleo previsto y de la inversión industrial en la provincia de Alicante y en la Comunidad Valenciana}

La evolución del empleo previsto y de la inversión, presenta las siguientes características en Alicante:

6 JORDÁ BORRELL, Rosa María: La industria en el desarrollo del área metropolitana de Valencia, Universidad de Valencia, Secció de Geografia, Alboraya, 1986, 302 p. 


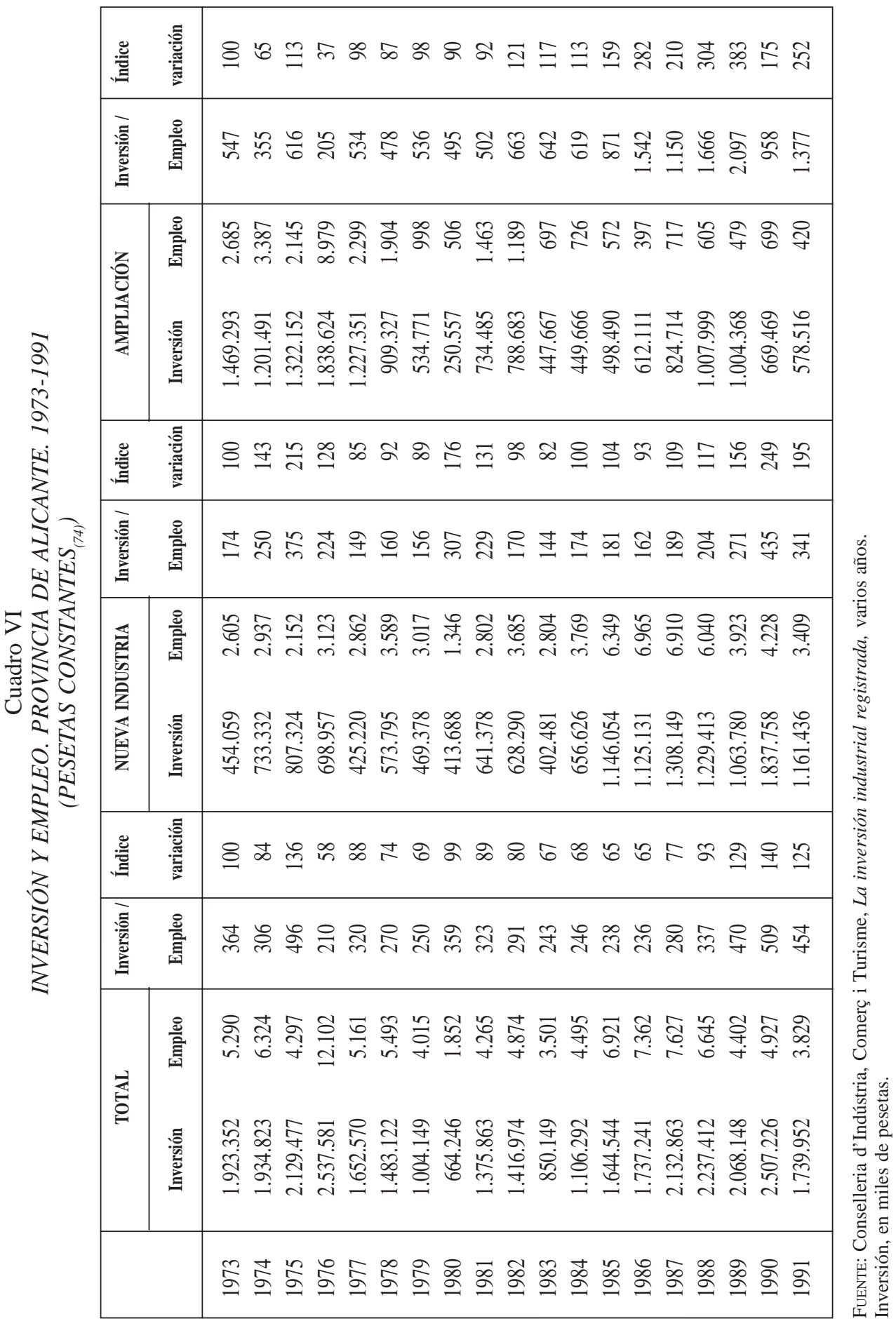




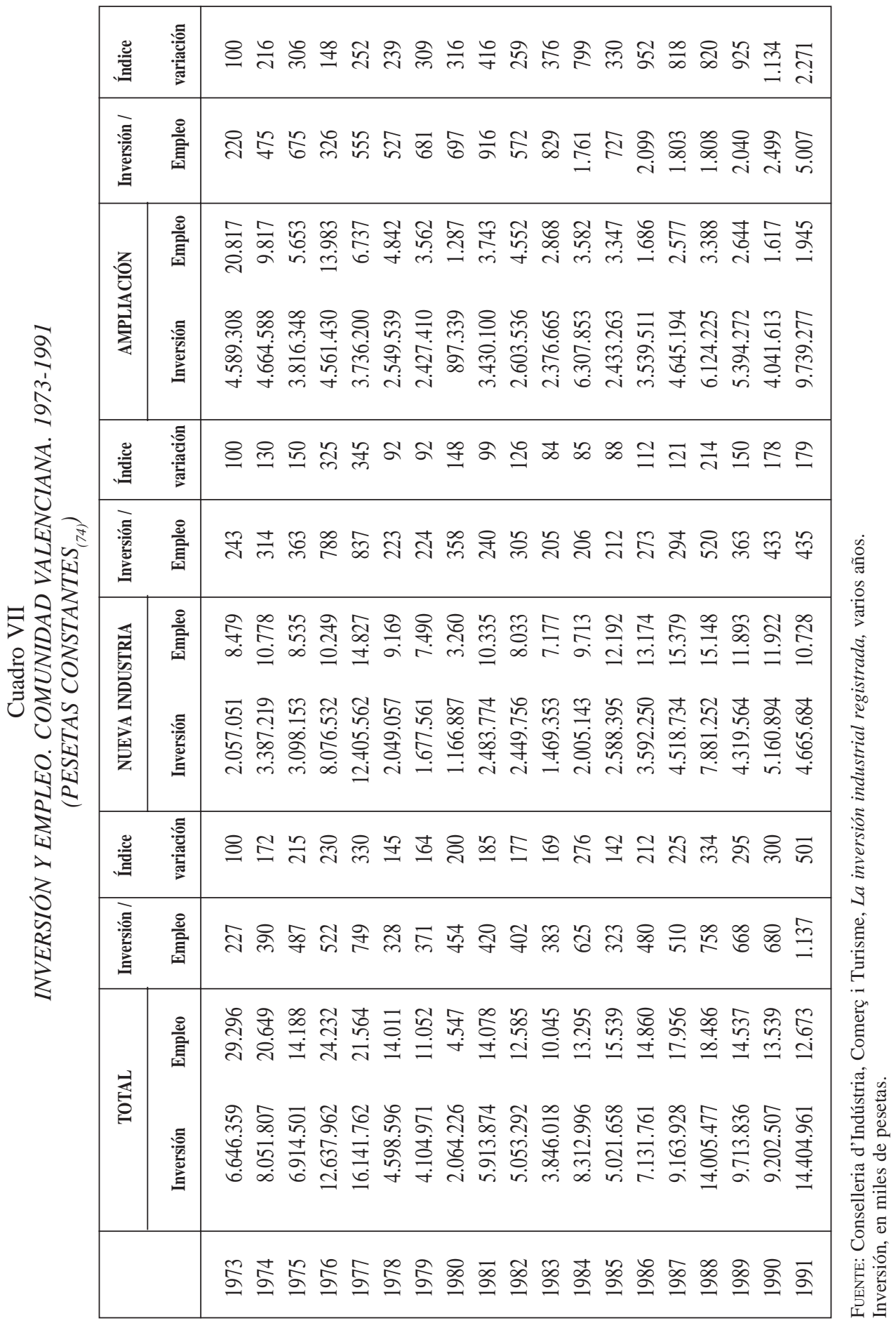


1.- La inversión industrial/empleo previsto, en pesetas constantes ${ }_{(74)}$ siguió una trayectoria regresiva en la provincia de Alicante desde 1973 a 1986, pero a partir de 1987 se recuperó, de modo que en 1991, como señala el índice de variación, superaba en un 25\% la de 1973, (Cuadro VI).

2.- - La inversión industrial-empleo previsto tampoco se distribuyó por igual entre las inscripciones en Nueva Industria y en Ampliación. Excepto en 1976, siempre se ha necesitado más dinero por empleo en las inscripciones de ampliación que en las de nueva industria; con una tendencia general a aumentar esta diferencia.

$\mathrm{Al}$ objeto de comprobar a otra escala la evolución de los resultados provinciales se ha realizado un análisis comparativo con los del conjunto de la Comunidad Valenciana, (Cuadro VII), estableciéndose las siguientes observaciones:

Existen coincidencias entre los dos espacios en cuanto a que las inversiones industriales más cuantiosas por empleo se producen en mayor medida en la ampliación que en la nueva industria, y en la tendencia a incrementarse las diferencias debido al menor crecimiento de la nueva industria. Las diferencias más relevantes son básicamente:

- Una mayor inversión media industrial por empleo previsto en la Comunidad Valenciana que en Alicante, que se muestra intensificada por la trayectoria seguida por la relación inversión-empleo, bastante más destacable también en la primera.

- La evolución de la inversión/empleo en las inscripciones de nueva industria es similar, por el índice de variación, en los dos espacios, sin embargo los valores absolutos continúan siendo menores para Alicante.

- La evolución de la inversión/empleo en las ampliaciones, marca la tendencia general, y es en este tipo de inscripciones donde existe mayor divergencia en los valores. De este modo, cabe señalar que en Alicante, el capital que se necesitaba para crear un puesto de trabajo era de 364.000 pts. constantes ${ }_{(74)}$ en 1973 y de 454.000 pts. en 1991, mientras que en la Comunidad Valenciana se pasó de 227.000 pts. constantes ${ }_{(74)}$ a 1.137 .000 pts. durante los mismos años.

Estos datos conducen a establecer la diferenciación de dos modelos industriales según el momento histórico y el espacio geográfico, como corresponde a espacios con sectores y procesos industriales específicos y no coincidentes.

En los primeros años de los setenta se puede distinguir en Alicante un modelo industrial más intensivo en capital que en el conjunto de la Comunidad Valenciana; al final de los ochenta la situación es la contraria. No obstante en los dos espacios, y especialmente en la Comunidad Valenciana, la evolución está definida por una mayor demanda de capital por puesto de trabajo previsto crear.

\section{La distribución geográfica del empleo previsto durante 1975 y 1991}

Hasta ahora, la localización industrial se ha estudiado estadísticamente; para completar esta investigación, la representación cartográfica permitirá profundizar en esta cuestión.

En primer lugar, se ha de destacar que, aunque el volumen de empleo industrial previsto retrocede entre 1975 y 1991, el número de municipios donde se genera empleo aumenta, pues se pasa de 63 a 73 .

En segundo lugar, se señala el cambio del modelo de concentración del empleo pues en 1975 había más municipios que superaban el 5\% del total del empleo industrial provincial (Alcoi, Alicante, Crevillent, Elx, Elda, Ibi, Verger), mientras que en 1991 en esta categoría sólo están Alicante, Crevillent y Elx, destacando la concentración de este último municipio que se aproxima casi a la cuarta parte del total provincial (24'3\%). 

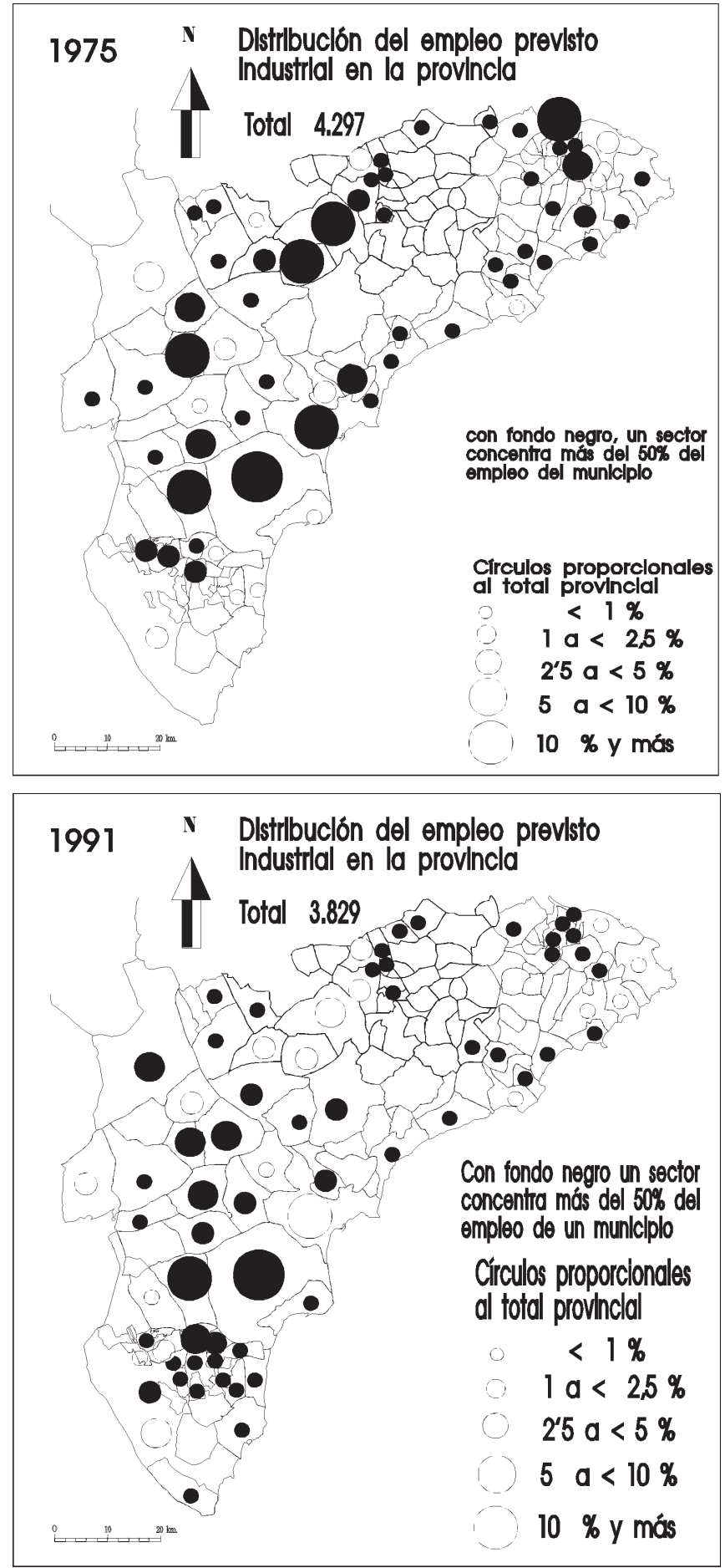
En tercer lugar, resulta evidente, en lo referente a la concentración sectorial del empleo en los municipios, la especialización municipal y la dependencia del empleo y de la economía de los municipios de los avatares de un sólo sector industrial (color negro en los círculos de los mapas). La especialización sectorial en los municipios se relaciona más con la industria del calzado, pues la mayoría de los círculos negros se sitúan en las proximidades de Elx, tanto por la Vega Baja, como por el Valle del Vinalopó.

En cuarto lugar, se observa que la diversificación se está produciendo en espacios con características diferentes: en el norte de la provincia tanto en su interior, donde existe una tradicional industria textil, y una importante y concentrada industria del juguete, y en el litoral, como consecuencia del creciente mercado que potencia el turismo.

En quinto lugar, se anota la existencia de un «desierto» en la creación de empleo en lo que se podría denominar «serranía de Aitana», y otro «semidesértico» durante 1975 localizado al sur de la provincia (Vega Baja del Segura), pero que afecta a pocos municipios y que en 1991 prácticamente ha desaparecido. En esta última zona, se ha producido además un fenómeno de concentración sectorial, pues en 1975 en el mapa los círculos que figuran son la mayoría de fondo blanco, mientras que en 1991 lo son de fondo negro. La explicación se halla en la difusión de un sector eminentemente manufacturero como es el del calzado. 